\title{
Insights into the Defect Structure Resulting from the Hydrogen Absorption in Palladium Nanocubes Using Liquid Cell Transmission Electron Microscopy
}

\author{
Sophia Betzler ${ }^{1}$, Colin Ophus ${ }^{2}$ and Haimei Zheng ${ }^{1}$ \\ ${ }^{1}$ Lawrence Berkeley National Laboratory, United States, ${ }^{2}$ Lawrence Berkeley National Laboratory, California, \\ United States
}

Efficient hydrogen storage technologies are essential to replace fossil fuels in the future. Ideal storage materials provide fast absorption/desorption kinetics and complete reversibility of the hydrogen absorption reaction. Palladium is well known for its ability to absorb hydrogen in interstitial lattice sites: at low hydrogen pressures a small hydrogen concentration is absorbed $\left(\alpha-\mathrm{PdH}_{0.03}\right.$ phase), higher pressures induce a first-order phase transition to $\beta-\mathrm{PdH}_{\mathrm{x}}$. The latter phase transition to a hydride system is accompanied by a $3.2 \%$ lattice expansion [1]. The localized swelling of the crystal lattice has dramatic effects on the atomic structure of the material which must accommodate these changes. Liquid cell transmission electron microscopy enables the direct observation of chemical reactions in real-time with atomic resolution. The interaction of the electron beam with water yields hydrogen radicals and molecular $\mathrm{H}_{2}$ [2]. The concentration of these radiolysis products depends on the dose rate and thus can easily be manipulated during the reaction.

In this study we exploit the radiolysis products to analyze the impact of the localized phase transition to $\beta$ $\mathrm{PdH}_{\mathrm{x}}$ on the atomic structure of palladium nanocubes. The lattice mismatch between the two phases reflects in dark fringes visible in bright field images recorded during the reaction (fig. 1b). In agreement with previous gas phase studies [3] the phase transition is initially localized at one corner of the nanocube before continuing through the rest of the cube. Atomic resolution images recorded during the reaction provide important insights into the propagation of the phase transition and we observe two different mechanisms. Fast hydrogen absorption causes a rapid phase transition with a sharp interface between the two crystal phases which moves in [100] direction through the lattice (fig. 2a). This rapid reaction yields a single crystalline $\beta-\mathrm{PdH}_{\mathrm{x}}$ nanocube with few defects. Slower reaction kinetics, on the other hand, result in an incomplete phase transition. After initially being localized to one corner, the phase transition does not propagate through the material; instead, the surface layer of the nanocube transforms to $\beta-\mathrm{PdH}_{\mathrm{x}}$. The lattice mismatch between the core and $\beta-\mathrm{PdH}$ shell causes significant strain within the cube which is compensated by a complex defect structure. This defect structure seems to affect the reversibility of the absorption reaction. While we observe the release of hydrogen for defect-free $\beta-\mathrm{PdH}_{\mathrm{x}}$, no significant changes of the crystal lattice are observed for defective nanocubes. These results suggest that the defect structure formed for incomplete phase transitions impedes the hydrogen release which might impact the life-time of functional devices.
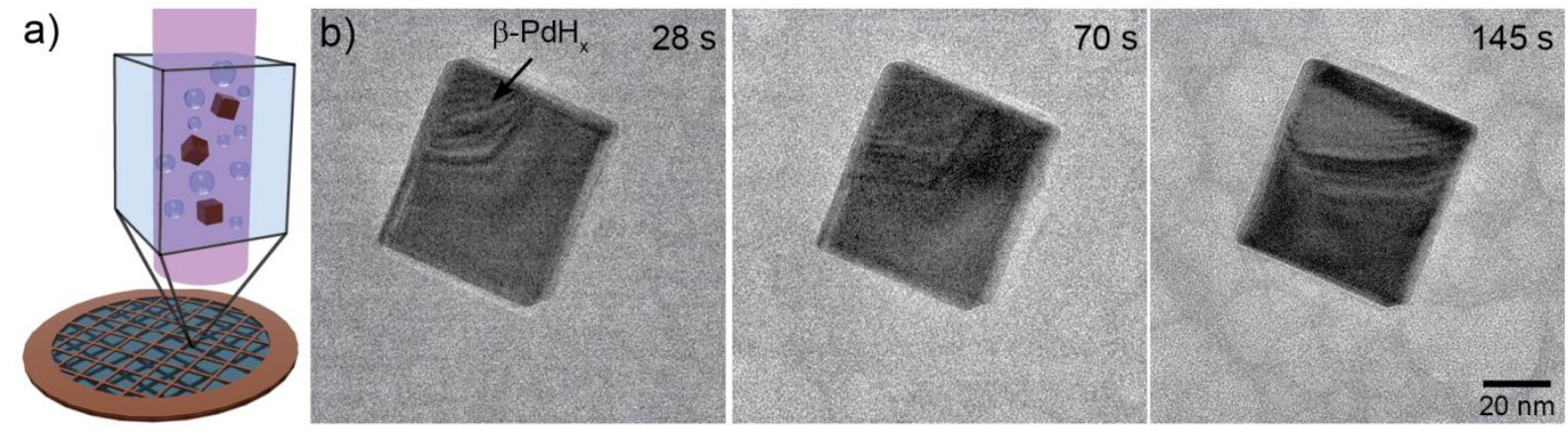

Figure 1. Liquid cells are used to study the absorption of $\mathrm{H} 2$ into palladium nanocubes. a) Radiolysis of water by the electron beam yields $\mathrm{H} 2$ and hydrogen radicals. b) Bright field images recorded during the experiment show the presence of two crystal phases within the nanocubes. The lattice mismatch between palladium/ $\alpha-$ 
PdH0.03 and $\beta$-PdHx causes black fringes in bright field images. The indicated time refers to the start of the beam exposure of this nanocube.
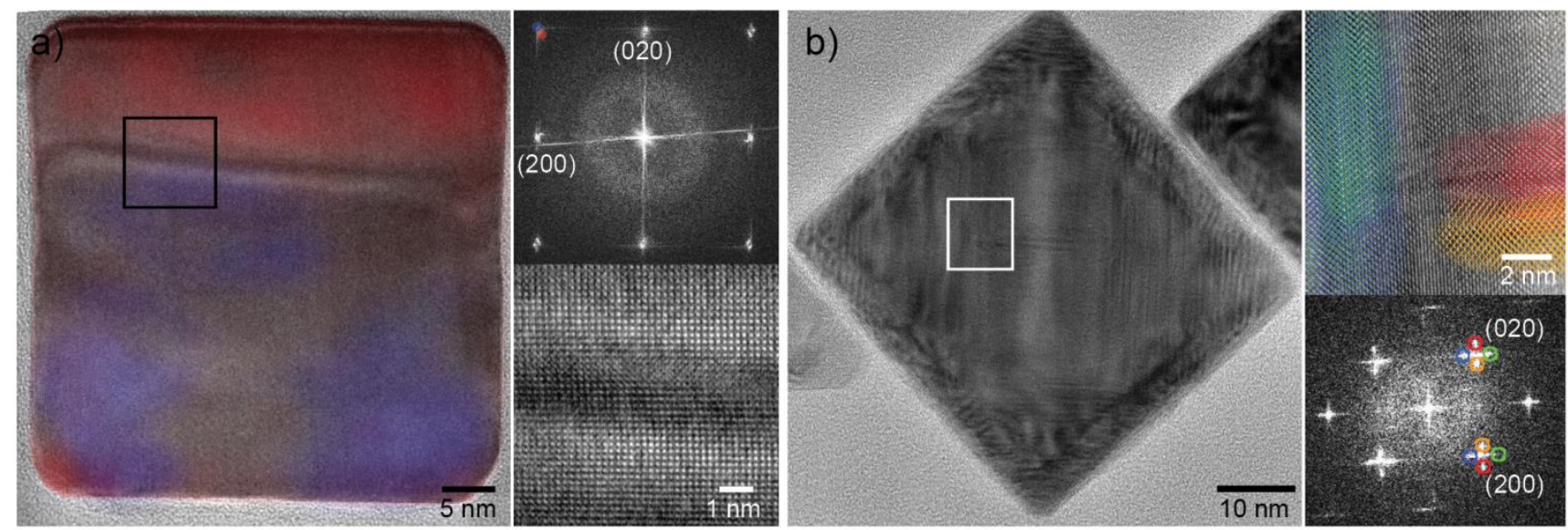

Figure 2. High resolution image of palladium nanocubes recorded during the phase transition of palladium nanocubes to $\beta$-PdHx. a) High resolution image and the respective Fast Fourier transformation (FFT) pattern recorded demonstrating a sharp interface between the two phases. The presence of two crystal phases is reflected by the splitting of the reflections in the FFT pattern. Bragg filtering was applied to highlight the two phases in the image (red: $\mathrm{PdHx}$, blue $\mathrm{Pda}-\mathrm{PdH} 0.03$ ). The inset shows the atomic structure at the interface between the two phases. b) Slower reaction kinetics result in an incomplete phase transition yielding a coreshell structure, with a hydride shell surrounding the core. The lattice mismatch between the two phases causes strain inside the cube which is compensated by a complex defect structure. The defect structure reflects in the peak splitting in the FFT pattern with the different spots correlating with grains localized around the defects inside the nanocube.

\section{References}

[1] Dekura, Shun, et al. "Hydrogen in Palladium and Storage Properties of Related Nanomaterials: Size, Shape, Alloying, and Metal-Organic Framework Coating Effects." ChemPhysChem 20.10 (2019): 1158-1176.

[2] Schneider, Nicholas M., et al. "Electron-water interactions and implications for liquid cell electron microscopy." The Journal of Physical Chemistry C 118.38 (2014): 22373-22382.

[3] Narayan, Tarun C., et al. "Direct visualization of hydrogen absorption dynamics in individual palladium nanoparticles." Nature communications 8.1 (2017): 1-8. 